At the same time the electron is in equilibrium with the metal cations and the neutral metal atoms according to the equation

$$
\mathrm{II}^{+}+\xi^{-}=\mathrm{MI} \equiv \text {, }
$$

where $\mathrm{M}^{+}$signifies the cation and $\mathrm{Mr}$ the neutral atom.

Buston, July I 4 , igos.

\title{
ON THE DISSOCIATION PRESSURES OF CERTAIN OXIDES OF COPPER, COBALT, NICKEL AND ANTIMONY.
}

H. W. Foote AND F. K. SMITH.

Received June $25,1908$.

In this article we wish to give the results obtained in measuring the dissociation pressures of certain oxides of copper, cobalt, nickel and antimony at high temperatures.

When an oxide decomposes reversibly into oxygen and a lower oxide or a metal, the dissociation pressure is a function of the temperature and independent of the relative amounts of the solids. The problem of measuring dissociation pressures consists, then, in determining the temperature of the oxide, and the pressure of the oxygen evolved.

The form of apparatus used in the work is shown in Fig. I. It is not drawn to scale. The oxide was placed in the boat $a$ of platinum or

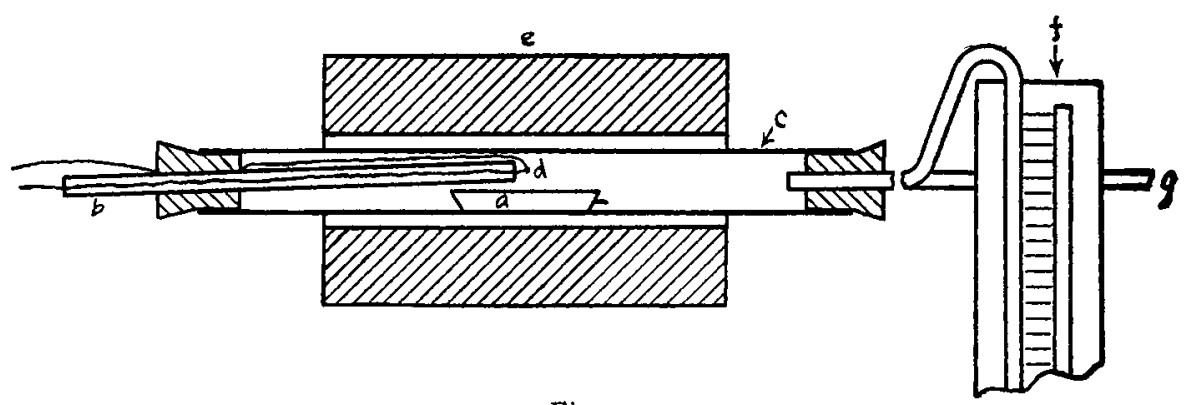

Fig. I.

porcelain. The boat was in the center of a porcelain tube about $60 \mathrm{~cm}$. long, and $15 \mathrm{~mm}$. inner diameter, which was heated by an Heraeus platinum resistance furnace $e$. The temperature was measured by a platinum and platinum-rhodium thermo-couple. The junction of the two wires was directly over the center of the boat at $d$. The wires were insulated from each other by a small quartz tube passing through a rubber stopper in one end of the porcelain tube. The latter projected far enough beyond the ends of the furnace so that the rubber stoppers did not become hot. The other end of the porcelain tube was connected with a mercury manometer $f$ by a glass tube passing through a rubber stopper. A branch of the glass tube connected with a pump is capable of lowering the pressure to less than a millimeter. The ends of the pyrometer wires 
were immersed in ice water, and then connected with a galvanometer reading temperatures directly. The pyrometer had been standardized by the maker. It was compared with another obtained at a different time, and was also checked by determining the melting point of zinc. We believe the temperature measurements are correct to within less than five degrees. Connections with the porcelain tube were made perfectly tight by covering the stoppers, and projecting quartz tube with sealing wax to which a little asphaltum varnish was added, to make it less brittle.

In carrying out an experiment, the boat and thermo-couple were placed in position, and the joints made tight. The apparatus was pumped out with a vacuum pump, and the glass tube connecting with the pump was sealed off. The furnace could then be heated, and temperatures and pressures read directly.

Cupric Oxide-Debray and Joannis ${ }^{1}$ have shown that cupric oxide on heating dissociates into cuprous oxide and oxygen, and that the action is reversible. They state that dissociation begins at a dull red, and that the pressure reaches $56 \mathrm{~mm}$. at the melting point of silver.

A number of temperature-pressure determinations were made, both with rising and falling temperature. Any temperature desired could be kept constant within one or two degrees by regulating the current heating the furnace. The pressures at any given temperature became constant in a surprisingly short time, usually within ten minutes. The values observed with falling temperature were slightly higher than those observed with rising temperature, as was perhaps to be expected. The pressures were determined using pure ignited cupric oxide, and also mixtures of cupric oxide and cuprous oxide, and found nearly identical. The mixtures of the two oxides were made from cupric oxide by pumping out part of the oxygen when the oxide had been heated till it began to dissociate. The results obtained were as follows. They represent the average of several sets of determinations with both rising and falling temperature.

$\begin{array}{cc}\text { Temperature. } & \text { Pressure (mm. of mercury). } \\ 900^{\circ} & \text { I } 5.8 \\ 950^{\circ} & 37.5 \\ 1000^{\circ} & 99 \\ 1010^{\circ} & 121 \\ 1020^{\circ} & 143 \\ 1030^{\circ} & 17 \mathrm{I} \\ 1040^{\circ} & 204 \\ 1050^{\circ} & 239\end{array}$

The results are plotted in Fig. 2.

Assuming that the pressure of the oxygen in the air is approximately I60 $\mathrm{mm}$., the dissociation pressure of the cupric oxide will reach this

' Compt. rend., 99, 583, 688 (1884). 
value at about $1025^{\circ}$, so that at temperatures above $1025^{\circ}$, cupric oxide becomes unstable, if heated in the air, and cuprous oxide becomes the stable form.

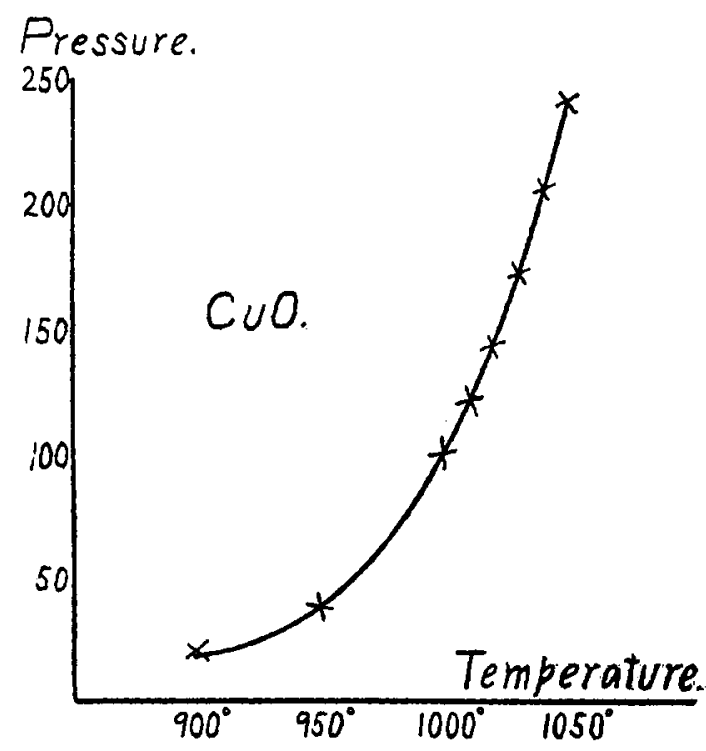

Fig. 2.

The mixtures of cuprous and cupric oxides obtained melted at approximately $1070^{\circ}$, as we determined in one experiment, so that the range of temperature where solid $\mathrm{Cu}_{2} \mathrm{O}$ is stable when heated in the air is only between $1025^{\circ}$ and about 1070.

Cuprous Oxide.-Pure precipitated cuprous oxide was heated in the apparatus previously described to about $1020^{\circ}$. The dissociation pressure did not exceed $\mathbf{I} \mathrm{mm}$. at this temperature, and further experiments on this oxide were discontinued.

Cobalto-Cobaltic Oxide, $\mathrm{Co}_{3} \mathrm{O}_{4}$.-Moissan states ${ }^{1}$ that when $\mathrm{CoO}$ is heated in the air it oxidizes to $\mathrm{Co}_{3} \mathrm{O}_{4}$, which is stable at $1000^{\circ}$ but breaks down into $\mathrm{CoO}$ if heated over the blast lamp.

Pure $\mathrm{CO}_{3} \mathrm{O}_{4}$ was made by precipitating potassium cobaltic nitrite, decomposing with sulphuric acid, precipitating the solution with sodium carbonate, washing free of sulphates and alkali and igniting cautiously over a Bunsen burner. Analysis showed that the residue was pure $\mathrm{Co}_{3} \mathrm{O}_{4}$, although for our purposes the presence of some lower oxide was not objectionable. The analysis of this compound was made by igniting it in a current of dry hydrogen and weighing metallic cobalt. We found 73.59 per cent. of cobalt while 73.44 per cent. is the calculated value.

1 Ann. Chem. [5], 21, 199. 
The following results were obtained: They represent the average of several series of observations made on rising and falling temperatures, using either pure $\mathrm{Co}_{3} \mathrm{O}_{4}$ or a mixture of this oxide with cobaltous oxide, obtained by pumping out part of the oxygen from the higher oxide, when it had begun to dissociate.

Temperature.
$800^{\circ}$
$850^{\circ}$
$900^{\circ}$
$910^{\circ}$
$950^{\circ}$
$970^{\circ}$

Pressure (mm. of mercury)
10
28
144
183
521
765

The results are plotted in Fig. 3. The dissociation pressure of the oxide reaches the pressure of the oxygen in the air at about $905^{\circ}$, so that above this temperature $\mathrm{Co}_{3} \mathrm{O}_{4}$ becomes unstable in the air, and forms cobaltous oxide, and oxygen.



Fig. 3.

Cobaltous Oxide.-To prepare cobaltous oxide, the boat was filled with cobalto-cobaltic oxide, and heated to about $970^{\circ}$, at which temperature the oxygen was repeatedly pumped off, until the pressure suddenly dropped, indicating that the cobalto-cobaltic oxide had been almost completely broken up into the monoxide. Analysis proved that the residue in the boat was very nearly pure cobaltous oxide. The boat was again placed in the furnace, and heated to about $110^{\circ}$, a pressure of 35 $\mathrm{mm}$. being observed. On repeatedly heating and evacuating, the pressure each time became less, and finally reached zero.

The pure cobaltous oxide thus obtained was heated at $I 100^{\circ}$ for three hours, no pressures being shown. Analysis again showed that no dis- 
sociation of the cobaltous oxide had taken place, as the percentage of cobalt was identical with that obtained before this prolonged heating. This experiment, together with the preceding ones, is not in agreement with the statement of Richards and Baxter" "that cobaltous oxide also at about $800^{\circ}$ dissociates very considerably into cobalt and oxygen."

The pressures which they obtained are easily explained by the results of our preliminary experiments, in which, as was noted above, considerable pressures were given, either by smaller amounts of the higher oxides, or by occluded oxygen.

Nickel Oxides.--Nickelic hydroxide was precipitated with bromine and sodium hydroxide, thoroughly washed, dried, and heated for some hours at $45^{\circ}$ in a current of oxygen. The resulting oxide, on analysis, was found to be practically pure nickelous oxide $\mathrm{NiO}$. This oxide, heated in oxygen, at temperatures varying from $200^{\circ}$ to $450^{\circ}$ did not change in composition.

It appears, then, that any action by which the higher oxide breaks down into nickelous oxide cannot be reversible, unless possibly at a high pressure.

Nickelous Oxide.-Nickelic hydroxide was ignited in the air, to convert it into nickelous oxide. The residue was heated in a vacuum, and thoroughly exhausted with the pump, to make certain that no trace of higher oxide was present. Preliminary determinations showed appreciable but variable pressures at about $1000^{\circ}$, but by repeatedly exhausting the tube with the pump, the pressure fell nearly to $0^{\circ}$, and remained there. This oxide, which may have contained a small amount of nickel, was heated like the other oxides, and the following results were obtained. But one set of observations was taken in this case.

$\begin{array}{cc}\text { Temperature. } & \text { Pressure (mm. of mercury). } \\ 800^{\circ} & 0.0 \\ 900^{\circ} & 0.0 \\ 1000^{\circ} & 2.0 \\ 1100^{\circ} & -5.5 \\ 1200^{\circ} & 13.0 \\ 1245^{\circ} & : 8.0\end{array}$

The heating, unfortunately, had to be discontinued at this temperature, as the porcelain tube had begun to soften, but the results are sufficient to show that dissociation had become very appreciable and that it is probably greater than the dissociation of cobaltous oxide.

Antimony Tetroxide.-This oxide was prepared by treating a hydrochloric acid solution of antimony trichloride with ammonia, filtering, and evaporating repeatedly with concentrated nitric acid. The residue was filtered, washed free from chlorides, and ignited to constant weight. 
We were unable to determine the dissociation pressures of this oxide accurately, for when dissociation began, the trioxide sublimed, and complicated the results. However, by heating a weighed quantity of the tetroxide in an open tube at various temperatures, and determining the lowest temperature at which loss of weight occurred, we were able to determine very closely the temperature at which the dissociation pressure of antimony tetroxide equals the pressure of the oxygen in the air. A series of such determinations showed that loss in weight first took place at about $950^{\circ}$. In determining antimony as tetroxide, therefore, it is possible to heat up to this temperature in the air, without loss of weight, provided reducing gases are excluded.

The dissociation pressures of a number of oxides have recently been calculated by Stahl ${ }^{1}$ on the basis of the Nernst formula connecting the dissociation pressure with heat of reaction, and absolute temperature:

$$
\log p=-\frac{Q}{4.57 \mathrm{~T}}+\mathrm{I} .75 \log \mathrm{T}+2.8 \text {. }
$$

The only results which can be compared with ours, are those on the dissociation of cupric oxide into cuprous oxide and oxygen, and of nickelous oxide into nickel and oxygen. In both these cases, the pressures calculated by Stahl are very much lower than the direct determinations made by us. This can be shown in the following summary:

Dissoctation of Cupric OXIDE.

\begin{tabular}{|c|c|c|}
\hline Temperature. & $\begin{array}{c}\text { Pressure } \\
\text { (Foote \& Smith). }\end{array}$ & $\begin{array}{c}\text { Pressure (mm.) } \\
\text { (calculated by Stah1). }\end{array}$ \\
\hline $900^{\circ}$ & I 5.8 & $\ldots$ \\
\hline $927^{\circ}$ & $\ldots$ & 0.020 \\
\hline $950^{\circ}$ & 27.5 & $\ldots$ \\
\hline $1000^{\circ}$ & 99. & $\ldots$ \\
\hline $1020^{\circ}$ & 143. & $\ldots$ \\
\hline $1027^{\circ}$ & $\cdots$ & 0.22 \\
\hline $1030^{\circ}$ & 171. & $\ldots$ \\
\hline & NICKEL OXIDE. & \\
\hline $1100^{\circ}$ & 5.5 & $\ldots$ \\
\hline $1127^{\circ}$ & $\ldots$ & $0.0,33$ \\
\hline $1200^{\circ}$ & 13.0 & $\ldots$ \\
\hline $1227^{\circ}$ & $\ldots$ & 0.0866 \\
\hline $\mathrm{I} 245^{\circ}$ & 18.0 & . \\
\hline
\end{tabular}

We found the temperature at which cupric oxide becomes unstable in the air, to be $1025^{\circ}$, while the calculated value obtained by Stahl is $1406^{\circ}$. We were unable to determine the exact temperature at which nickel oxide becomes unstable, but judging by our results, the temperature calculated by $S t a h 1\left(247^{\circ}\right)$ is certainly very much too high.

The dissociation pressures calculated by Stahl for low temperatures are so exceedingly small that they should be mentioned. Thus, the 
calculated pressure in atmospheres for nickel oxide at $227^{\circ}$, is $1.859 \times 10^{-45}$ and for zinc oxide, $1.333 \times 10^{-67}$.

Now the weight of a single molecule of oxygen is supposed to be not far from $7.36 \times 10^{-23}$ grams so that the gram molecular weight would contain $4.3 \times 10^{23}$ molecules. If the gas were under normal conditions, $0^{\circ} \mathrm{C}$. and $\mathrm{I}$ atmosphere pressure, the pressure exerted by a single

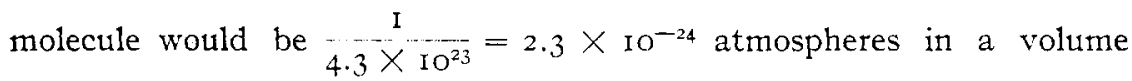
of 22.4 liters, or $4.1 \times 10^{-24}$ atmospheres at $227^{\circ}$. An equal volume of oxygen in equilibrium with nickel oxide at this temperature would exert a pressure, according to Stahl, of $1.849 \times 10^{-45}$ atmospheres, so that a single molecule shoud exert a pressure approximately $2 \times \mathrm{IO}^{2 \mathrm{I}}$ times as great as the oxygen in equilibrium with nickel oxide, a result which would appear impossible.

Summary.-(I) The dissociation pressures of certain oxides of copper, cobalt, nickel, and antimony, have been determined at different temperatures, and when possible, the temperature has been determined at which the dissociation pressure equals the pressure of the oxygen in the air.

Results on the dissociation pressures of cupric oxide and nickelous oxide have heen compared with results calculated by Stahl from the formula of Nernst. In both cases, the observed pressures are very much higher than those calculated.

SHEFFIELD LABORATORY, NEW HAVEN, CONN., May, Igos.

\section{ON THE DISSOCIATION PRESSURES OF FERRIC OXIDE.}

\section{BY P. T. WALDEN.}

Received June 25, Ig08.

As early as $1848 \mathrm{H}$. Rose $^{1}$ noticed that if ferric oxide were heated in a porcelain furnace there resulted a black residue which ground to a black powder and gave the well-known blue precipitate when its solution was treated with potassium ferricyanide.

By means of his optical pyrometer Le Chatelier² determined the temperature of the hard porcelain furnace to be $1370^{\circ}$. Evidently we may expect ferric oxide to begin to dissociate into oxygen and magnetic oxide at some temperature below $1370^{\circ}$ and the pressure of the oxygen at that point should be at least equal to the pressure of the oxygen of the air (160 mm.). Assuming that the above dissociation is reversible, the pressure due to the oxygen evolved should be a function of the temperature and independent of the amount of solid.

1. A. A. 74, 440.

${ }^{2}$ High-temperature Measurements, Le Chatelier and Boudouard, p. 220. 